\title{
Correction to: Interplay between Paper-and-Pencil Activity and Dynamic-Geometry-Environment Use during Generalisation and Proving
}

\author{
Kotaro Komatsu $^{1} \cdot$ Keith Jones ${ }^{2}$ \\ Published online: 5 June 2020 \\ (C) Springer Nature Switzerland AG 2020
}

\section{Correction to: Digital Experiences in Mathematics Education https://doi.org/10.1007/s40751-020-00067-3}

The original version of this article unfortunately contained a mistake. Figure 2 was initially published without the essential accompanying text. The corrected Fig. 2 does not change the findings or any other aspect of the paper.

The original article has been corrected.

Task 1. Put four points A, B, C, and D on circle $\mathrm{O}$, and let point $\mathrm{P}$ be the intersection point of segment $\mathrm{AC}$ and segment $\mathrm{BD} . \triangle \mathrm{PAB}$ and $\triangle \mathrm{PDC}$ can be made by respectively connecting $\mathrm{A}$ and $\mathrm{B}$, and $\mathrm{C}$ and $\mathrm{D}$. Prove that $\triangle \mathrm{PAB}$ and $\triangle \mathrm{PDC}$ are similar.

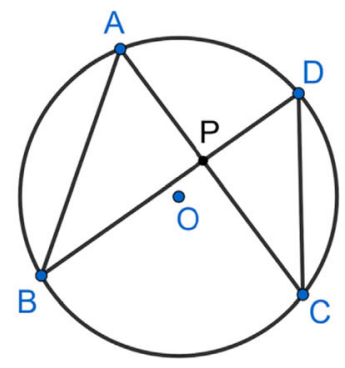

Fig. 2 Original statement

Publisher's Note Springer Nature remains neutral with regard to jurisdictional claims in published maps and institutional affiliations.

The online version of the original article can be found at https://doi.org/10.1007/s40751-020-00067-3

Keith Jones

d.k.jones@soton.ac.uk

1 Institute of Education, Shinshu University, 6-ro, Nishinagano, Nagano 380-8544, Japan

2 Southampton Education School, University of Southampton, University Road, Southampton SO17 1BJ, UK 